\title{
An investigation of a compact heat exchanger unit using CFD with experimental support
}

\author{
Pavel Peukert ${ }^{1, a}$, Jan Kolár ${ }^{1, b}$, and Karel Adámek ${ }^{1, c}$ \\ ${ }^{1}$ VÚTS a. s., Svárovská 619, 46001 Liberec, Czech Republic
}

\begin{abstract}
The paper contains a comparison of a numerical simulation with a real experiment. In the measured and simulated device is located a heat exchanger with a centrifugal fan. Due to the fan and the geometrical arrangement the flow is relative uneven and so the heat transfer hard to predict. The simulation should be time and cost affordable, so a standard $\mathrm{k}-\epsilon$ turbulence model and a relative simple mesh was used for the computations.
\end{abstract}

\section{Introduction}

Numerical simulations represent today a common method for to support the design process of new products. The decision to use such methods varies from cost and time saving to basic flow investigation in local areas where the experimental approach is often not possible.

The numerical simulations should go hand in hand with experimental validation, because the computations are given by a simplified mathematical description of a real physical system. The simplification of the system description is necessary for a time relevant computation with an affordable computation equipment. For common engineering tasks the DNS (Direct Numerical Simulation) is unusable so still the RANS (Reynolds-averaged Navier-Stokes equations) based turbulence models represents the main group. Especially the two equation models are popular. Often the various $\mathrm{k}-\epsilon$ and $\mathrm{k}-\omega$ models are usable for a lot of common tasks. A detailed explanation of the problematic could be find in [1] or [2].

This paper introduces a numerical flow simulation with heat transfer which was validated with experimental data. The main part is a heat exchanger with an uneven inlet profile, from the point of flow direction and velocity size. Because of the design complexity a high element count had to be used, but the detail resolution partially depend on the used computer performance so a balanced element size setting, modeling parameters had to be chosen. The experimental part should confirm proper model selection and settings sufficiently.

\subsection{Experimental device}

For experimental purposes was prepared a unit which is shown in 1 . The case holds all internal parts and defines the air flow path.

At unit the bottom is located a centrifugal fan, which sucks the air from the surroundings and transport the air to the heat exchanger. The air is heated up and exits the unit via an air grill, which gives the stream a certain direction.

\footnotetext{
a e-mail: pavel.peukert@vuts.cz

b e-mail: jan.kolar@vuts.cz

c e-mail: karel.adamek@vuts.cz
}

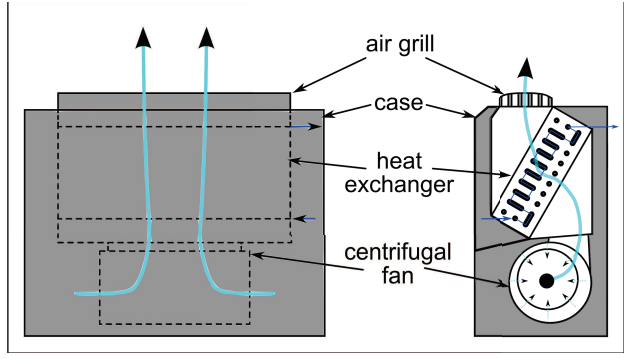

Figure 1. measured experimental unit.

The fan is a usual commercial one with few speed stages and a plastic impeller.

The heat exchanger could be classed as a compact cross flow tube-fin gas-liquid heat exchanger [3]. It consists from copper tubes and about 150 aluminum fins. A scheme of the side view is shown in 2 , with the water path and numbered tube passages. It is suggested that incoming air enters the heat exchanger under an angle, which is variable at different points of the surface. So the tube layout for the flow is mainly considered to be staggered.

The air grill is a simple grating with a modified outlet for the control of the outlet stream direction.

\section{Experiment description}

To get appropriate data for the validation of numerical simulations, two experimental parts were done. One consists of the velocity measurements of non heated air stream from the air grill and the second measures the heat transfer rates and temperature distribution of the experimental unit. The experiments were done at different fan speed levels, and water temperature inlets and flow rates.

\subsection{Velocity profile}

The measurements of the outlet velocity profile were done with hot wire anemometry. The hot wire probe was traversed automatic in a predefined grid in a specified height over the air grill. A simplified layout of the measurement is 


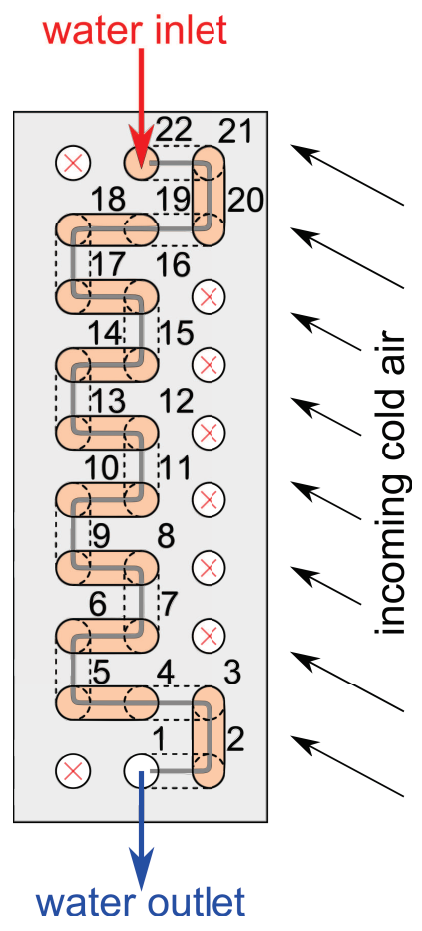

Figure 2. Heat exchanger description.

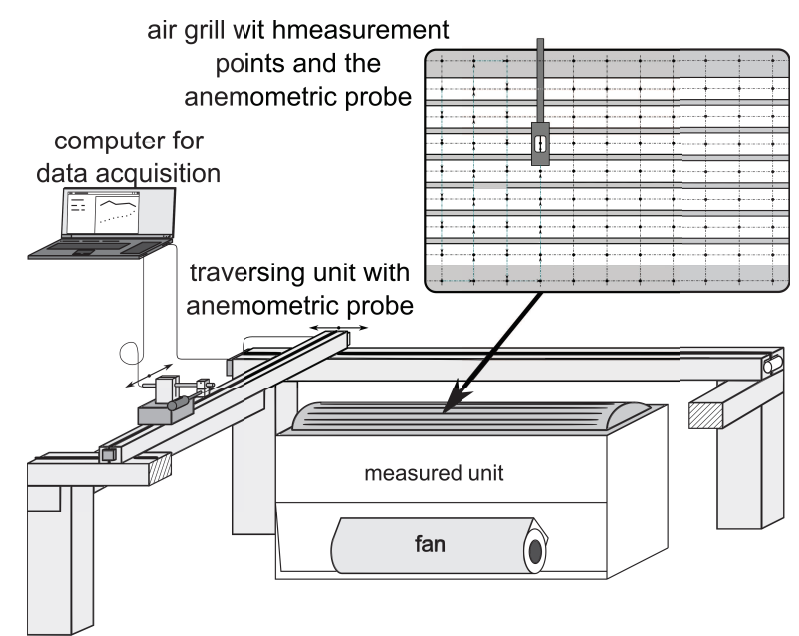

Figure 3. Measurement of the velocity profile at specified height above the air grill.

shown in 3 . The position and velocity data were collected to a computer for later evaluation. The measurements were performed in an empty and closed room to ensure minimal disturbances. To have an adequate compartment, the measurements were taken for all three speed levels of the fan.

With respect to probe (wire) dimensions was chosen a grid which places the measurement points of one column in the center of the slit passage, totally seven and two points outside of it. In the longitudinal direction were equally placed 33 points, where again the side point were not above the slots. The measurements were done in two planes above the air grill, the first at a height of $10 \mathrm{~mm}$ and the second at $100 \mathrm{~mm}$ from the highest point of the air grill.

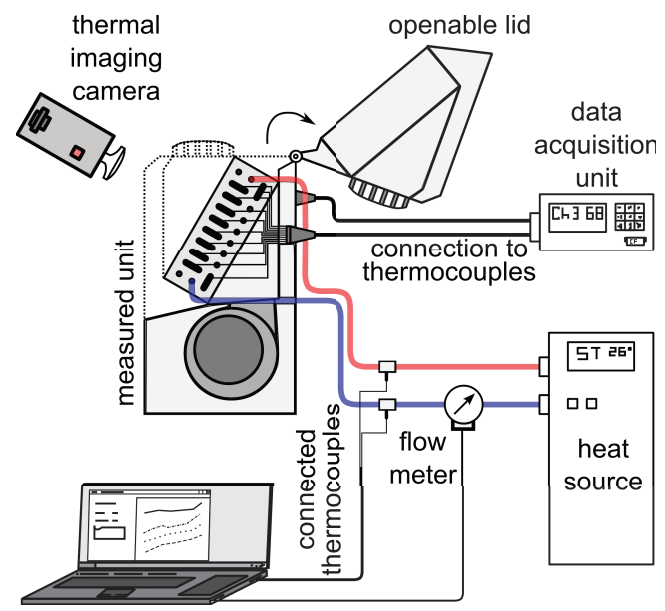

Figure 4. Experimental layout for heat transfer measurement.

\subsection{Heat transfer rates and temperature distribution on the heat heat exchanger surface}

The measurement was performed in an empty room with relative steady climatic conditions. The experimental layout is shown in 4.

The measured unit was prepared to provide the ability of fast lid removing to observe the temperature field on the heat exchanger with a thermal imaging camera. The heat exchanger was connected to a heat source via hoses. At the heat exchanger connections were special widgets with thermocouples for bulk temperature measurements to get the total water temperature gradient trough the heat exchanger. On one of the hoses was installed a flow meter to get the water flow rate.

On every knee, which connects two tubes was installed a thermocouple, like it is shown in figure 5. A very slight layer of thermal grease was applied between the thermocouple and the copper surface to minimize thermal resistance. An expected measurement error for calibrated thermocouples could be expected up to $0.2^{\circ} \mathrm{C}$. The data from the thermocouples were collected via a data acquisition unit on a flash disk for later evaluation. So it was possible to get the temperature drop on every tube, but due to uneven air flow profile trough the heat exchanger it was appropriate to get an idea about the distribution along tubes. For that reason was used the thermal imaging camera. A little problematic is to ensure to determine the right emissivity of the aluminum fins so a calibration measurement was done. The heat exchanger was placed in a climatic chamber. The temperature in the chamber was set to few temperatures, which ranged about the expected values and an averaged emissivity was calculated.

The whole measurement takes place in following way. The fan of the measured unit was set to a given level. A demanded water temperature was set to the inlet of the heat exchanger and the flow rate was changed until a required temperature drop between the inlet and outlet achieved. After the whole system was stabilized, the water flow rate, temperature on the inlet, outlet and individual knees was measured. Then one of the technicians opened fast the removable lid of the unit, while another take fast a picture of the actual temperature field of the heat exchanger. Then the whole process could start for another temperature. 


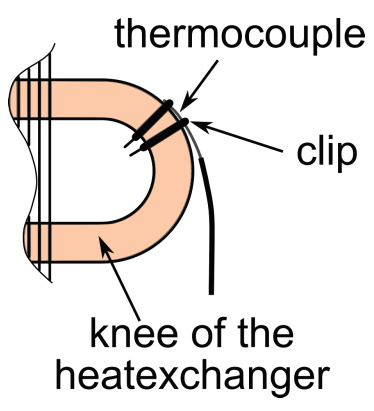

Figure 5. Heat exchanger knee with thermocouple.

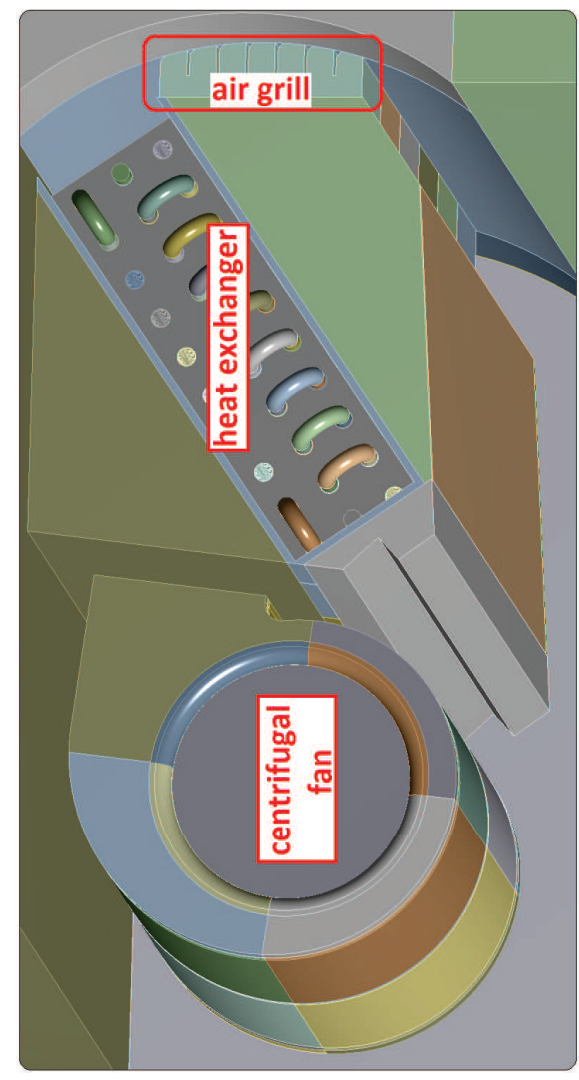

Figure 6. Geometrical model.

\section{Numerical simulations}

The preprocesing numerical simulation was done with the comercial software package Ansys Fluent.

\subsection{Model and Mesh}

The model of the measured unit was simplified, to substantial details, which noticeable effect the flow. In figure 6 is shown, the geometrical model. It consists from few zones, the centrifugal fan, the inner part of the unit, the heat exchanger, the air grill and the surroundings.

The model was meshed via Ansys Meshing and counts more than 10.5 million elements, a part of it is shown in figure 7. It is clear that the mesh and model was done with respect to a reasonable computation time. For example it was made a concession to the mesh size although a more detailed mesh were desired in the upper part with the unstructured tetrahedron zone in figure 7 , because here lies

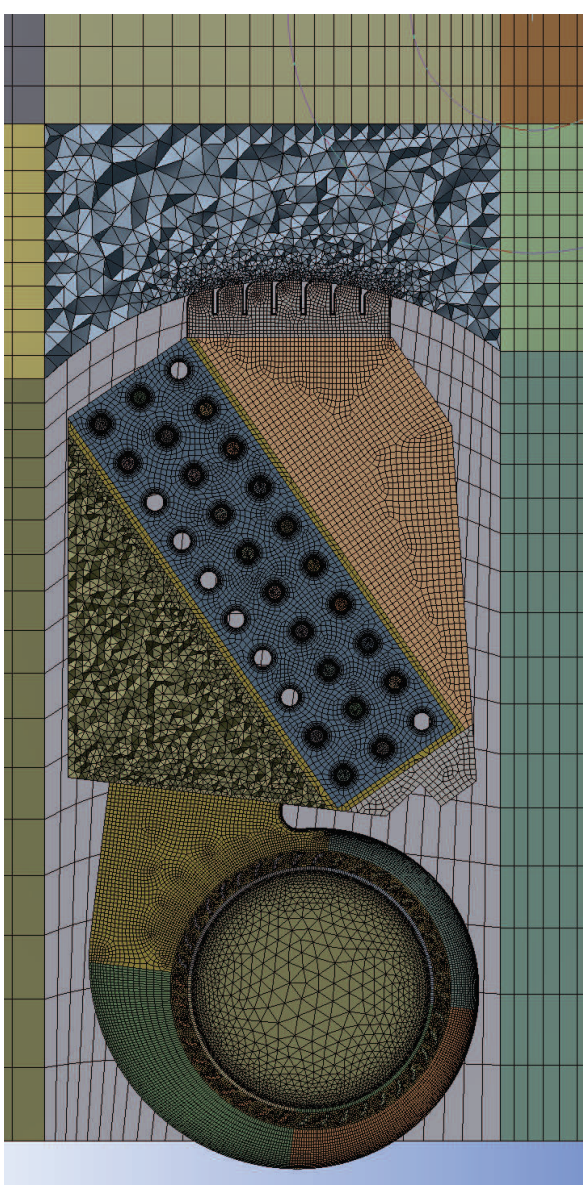

Figure 7. Computational mesh.

the second cross section where the velocity profile was measured.

The heat exchanger is the most complex part of the model. It takes about 6.5 million elements. The heat transfer was modeled directly from the heating water to the cold air. The heat exchanger model is relative detailed. It includes all tubes and fins. To ensure a good simulation of the heat transfer the mesh of the first fin was swept over the entire heat exchanger in the tube axis direction. The mesh in the air gaps between the fins is formed with a maximum grow rate factor of 1.2 so that in the middle is located the highest element and at the surface region were the smallest ones to allow a proper computation of the boundary layer effect. In the normal direction to the tube axis is the mesh relative coarse, but detailed enough due to the flow character despite the higher aspect ratio of the elements.

The centrifugal fan part is also relative complex. The geometry of the impeller blades is based on a contour scan of the impler where the geometry was measured. The fan model is divided in to three zones. The inner stationary zone, the moving reference frame zone, and the remaining fan housing zone.

Inner zones of the unit, copy coarsely the original geometry, such detail as small scree holes, small chamfers were omitted.

The geometry of the air grill is designed like the original, with radiuses and gaps.

The surroundings is few meters around the unit to ensure no affecting of the flow character in and a certain area around the unit. 


\subsection{Computations}

For processing of was used Ansys Fluent. Because the flow is over most zones turbulent, the standard k-epsilon model was used. The task was defined as a steady problem. To initiate the flow, the motion reference frame was set to the same impeller speed like it was determined in the experiment. The pressure inlet on the centrifugal fan was set with zero overpressure, like the pressure outlets on the surroundings boarder. The temperature of the incoming water to the heat exchanger was set to the measured temperature in the experiment and the mass flow rate also. Material properties were set corresponding to the real used materials or similar. The air was assumed like incompressible, with constant properties and density, which is acceptable due to the flow and temperature range. The task was initialized and run for few thousand iterations, until the energy residuals stagnate. The mass flow and energy balance was checked.

\section{Data evaluation}

The obtained data from the experiment and numerical simulations were brought together, evaluated and compared. Because of the large amount of data sets only a one representative set result will be shown. Some of the comparison sets which show colored contours don't use the same color scheme, because different software was used for evaluation (for the experimental part Wolfram Mathematica and for numerical Ansys Fluent).

For all measurements and simulations were tested 3 regimes of different fan speed levels and different temperature drops. Because these tests result in a large output set, only the second set on the middle fan speed level is presented. It should be mentioned, that that case represents the worst in the comparison between computation and measured values.

\subsection{Velocity profile comparison}

For the comparison of the velocity profile outlet of the unit was chosen to show the case with the third fan speed level. In figures 8 and 9 were shown the results of the measured and computed velocity profile $10 \mathrm{~mm}$ over the air grill. The pictures don't look direct the same, because the picture of the experiment had a significantly smaller resolution and averages over a larger area, the experimental data were interpolated for smoother transitions. But both figures had the same tendencies and similar velocities at the given points. So in 8 and 9 is the same profiles. The velocity decrease from the sides to the middle at the bottom part of the figure. And the highest velocities at the top. The velocity peaks from the measurement were about $2.8 \mathrm{~m} / \mathrm{s}$ and from the simulation about $2.7 \mathrm{~m} / \mathrm{s}$, which is comparable.

If the figure 10 and 11 is shown a cross section in the height of $100 \mathrm{~mm}$. If they were compared the tendency is relative similar, also something like the two bumps, could be observed in 10 but in the experiment holds the stream a more compact shape. That could be given due to the bigger elements, which grow fast behind the air grill. That could be seen in 7 . In the observed cross section, the elements had sizes comparable with the air grill gap, but it

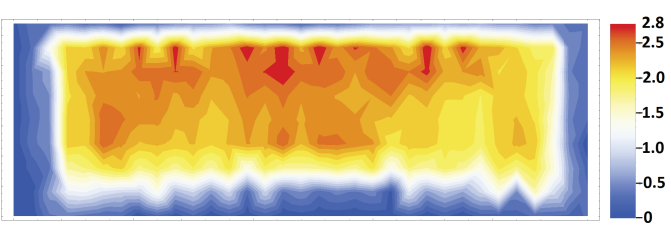

Figure 8. Measured velocity profile in height level $10 \mathrm{~mm}(\mathrm{~m} / \mathrm{s})$.

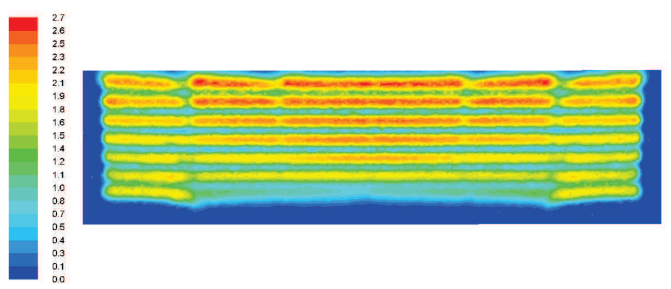

Figure 9. Computed velocity profile in height level $10 \mathrm{~mm}(\mathrm{~m} / \mathrm{s})$.

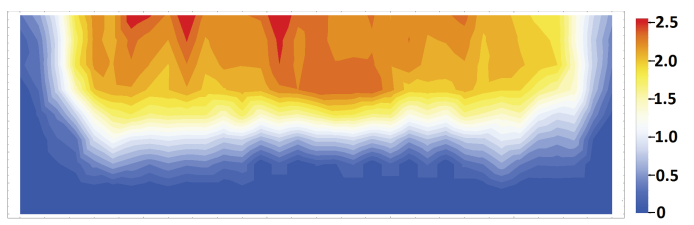

Figure 10. Measured velocity profile in height level $100 \mathrm{~mm}$ $(\mathrm{m} / \mathrm{s})$.

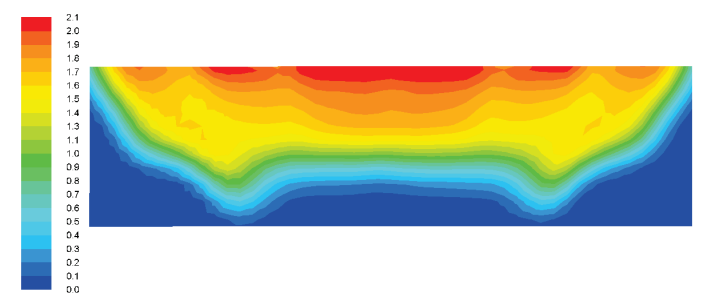

Figure 11. Measured velocity profile in height level $100 \mathrm{~mm}$ $(\mathrm{m} / \mathrm{s})$.

should not influent the desired phenomenon. But if the velocities at that cross section were the main observed theme, the mesh should be refined. The velocity peaks here were about $2.5 \mathrm{~m} / \mathrm{s}$ for the experiment in three points, but most of the time is the upper corner at velocities about $2.2 \mathrm{~m} / \mathrm{s}$. In the simulation the peaks at the upper corner is about $2.1 \mathrm{~m} / \mathrm{s}$, which quite similar.

For the not shown results the comparison was similar, and should be mentioned as satisfying.

\subsection{Temperature profiles on the heat exchanger}

To verify the heat transfer part of the simulation was chosen to observe the temperature distribution over the heat exchanger. In figure 13 is shown the picture taken by the thermal imaging camera (the lighter part are warmer than the dark ones), where the temperature across the drawn lines is depicted in figure 14. In comparison with figure 15 is the temperature measured via the camera lower about $5^{\circ} \mathrm{C}$, but the temperature pater is near the same, what supports the numerical results. To measure the temperature is relative complicated, especially for material like aluminum and if one presumes to look in to the gaps between the fins. 


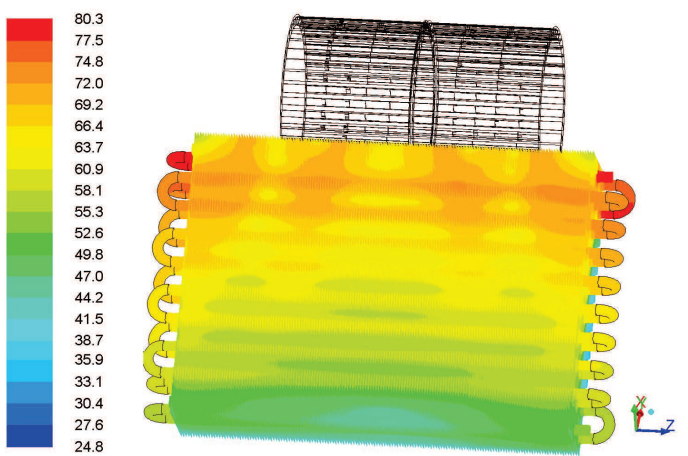

Figure 12. Computed heat exchanger temperatures.

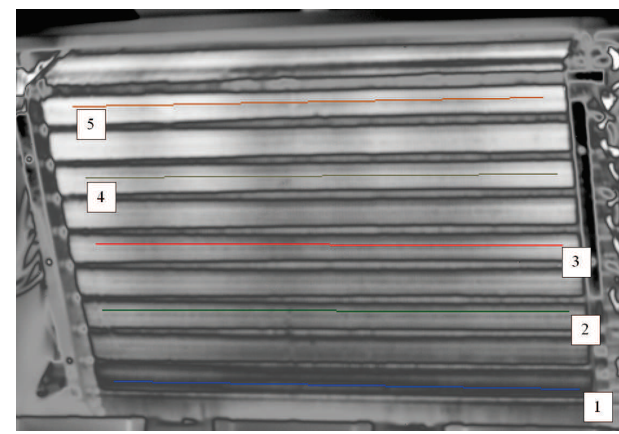

Figure 13. Heat exchanger image taken by thermal imaging camera.

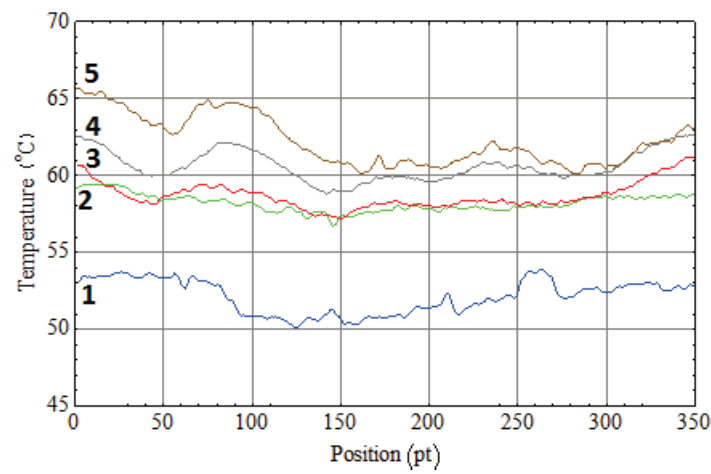

Figure 14. Temperature for given lines in figure 13.

Here could a slight change in the view angel change the absolute temperature.

\subsection{Temperature on knees and heat transfer performance}

A better way for direct temperature comparison is the knee temperature and the in and outlet water bulk temperature, like it is shown in figure 15. It should be mentioned, that the knee number 0 mean bulk outlet and 22 bulk inlet temperature, so their comparison to the other values should be done with care. The measured temperature shows a tendency how the temperature drops with further progress of the bulk. For points 2 and 5 could be expected a poor contact between the thermocouple and the knee surface, which was confirmed by other measurements too. At some points the temperature drop is near stagnant. That is easy to ex-

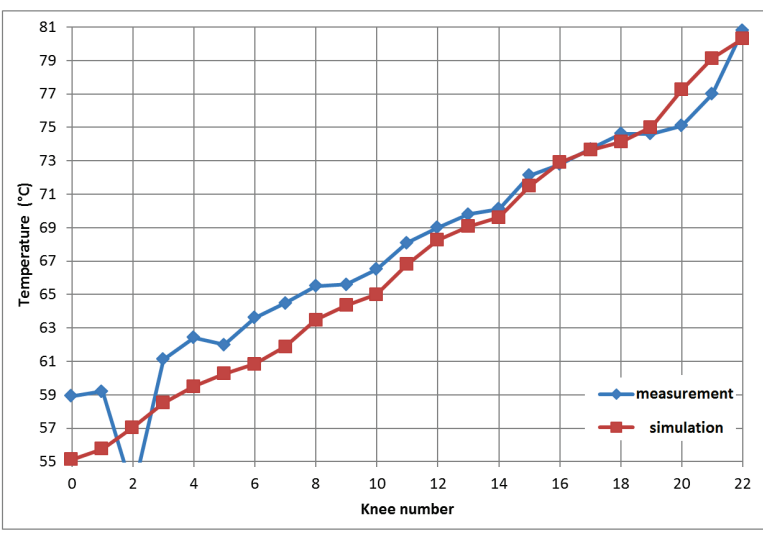

Figure 15. Chart of heat exchanger knees and in/outlet.

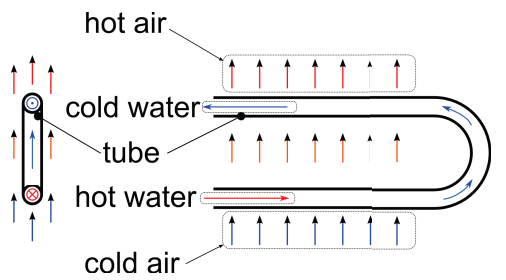

Figure 16. Cross counter flow arrangement.

plain, because some tubes are arranged near cross parallel flow due to the air flow, like it is show in figure 16. So that the warmer water is significantly cooled down by the incoming cold air and the air is heat up. For the second tube is the temperature difference between the air and water significantly smaller, so that the water temperature drop is relative small compared to the first tube. This case corresponds to the knee pairs 4-5, 8-9, 18-19. A little strange looks the pair 14-15, which has a relative high temperature drop, and between 13-14 is the decrease low. If the simulation is compared to the measurement, so there is often (excluded point 20 and 21) a similar tendency, but the heat transfer rate is clearly over predicted by the numerical part.

But the desired value is the heat transfer rate, which is computed by equation 1 , where $\dot{m}$ represents the water flow, $c_{\mathrm{p}}$ the specific water heat capacity and $\Delta t$ is the difference between the in and outlet water bulk temperature. For the presented case is the overestimation by the numerical simulation about $18 \%$.

$$
\dot{Q}=\dot{m} c_{\mathrm{p}} \Delta t,
$$

To investigate the case more precisely, from the aspect of heat transfer on individual tubes a better value is a percentage heat transfer rate on the given tube $\dot{Q}_{i}^{*}$ related to the total heat transfer rate $\dot{Q}_{\text {total }}$, which is given by equation 2.

$$
\dot{Q}_{\mathrm{i}}^{*}=\frac{\dot{Q}_{\mathrm{i}}}{\dot{Q}_{\text {total }}},
$$

Figure 17 shows a plot of the percentage heat transfer rates on every tube. The three lines represent the experimental, simulated and corrected experimental values. The corrected values take in account that the measured value was for sure wrong (that based also on additional measurements). So was taken a mean value computed by the temperature of the next two usable thermocouples and divided 


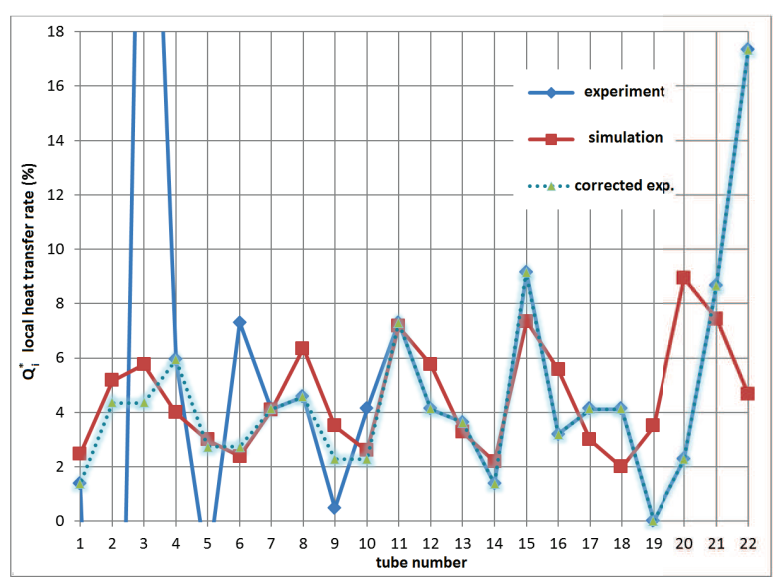

Figure 17. Local procentual heat transfer rate on individual tubes.

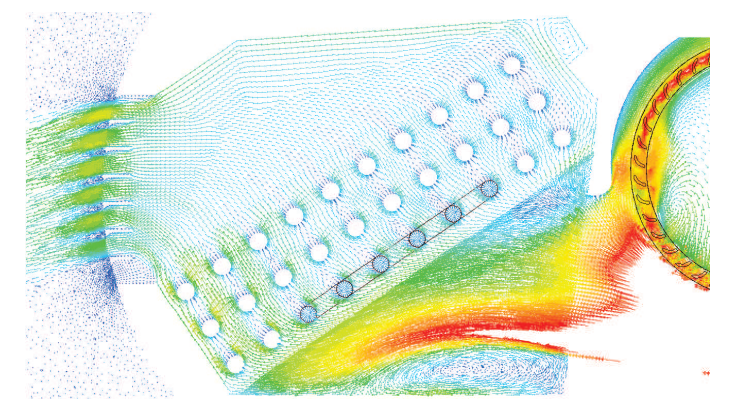

Figure 18. Vector array at $1 / 4$ of the units length.

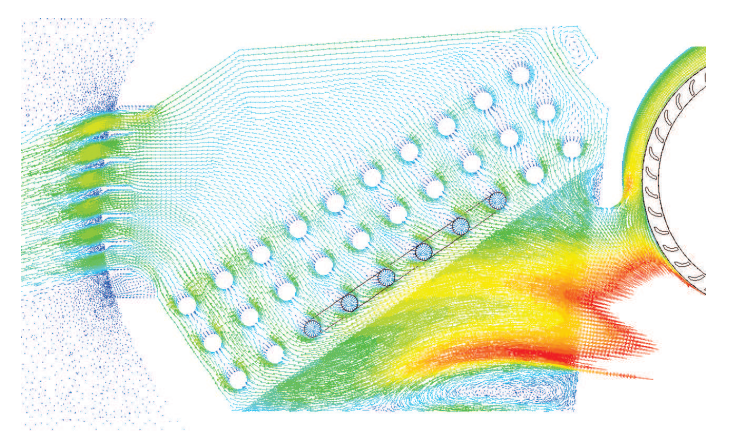

Figure 19. Vector array at $1 / 2$ of the units length.

by two, to have and approximate idea of the total influence of this part.

In figure 18 is shown a cross section trough $1 / 4$ of the heat exchanger witdth. It could be observed that the main part of the flow and the highest velocities go through the left bottom part. Here is also the flow arrangement of the tubes to the flow clearly aligned, while in the middle and the top of the heat exchanger is the flow tube arrangement more staggered. But one should be carefully with this interpretation, because if a look is taken to figure 19 (cross section in $1 / 2$ of the heat exchanger width) the flow changes, like it could be expected from figure 20. In figure 19 is the flow from the fan distributed much more evenly and with higher flow rates in the middle of the heat exchanger. Due to the varying air flow character and distribution over the heat exchanger the heat transfer prediction by a classic empirical method like it could be find in The Heat Exchanger Design Handbook [3].

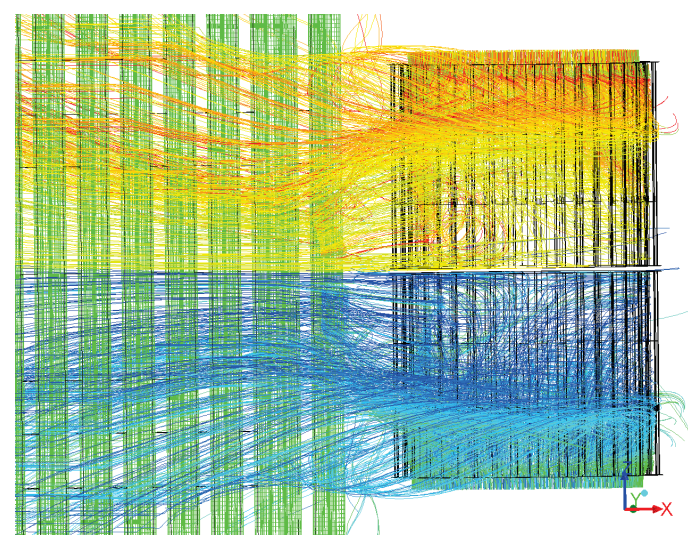

Figure 20. Pathlines colored by particle track.

The path lines in figure 20 even more support view of the uneven and diverse flow.

\section{Conclusions}

The comparison between the experiment and the numerical simulation had shown that the k-epsilon model in Ansys Fluent is a capable turbulence model for simulations with heat transfer in the mentioned conditions, even though the prediction is overestimated. Because for similar flow conditions the model could reveal new construction improvements to increase or decrease the heat transfer, depending on the need.

In this paper was introduced only one case, but three different fan setting were measured and simulated. The estimated heat transfer rates lies in a predicted field between 13 to 18 percent of overestimation compared with the measured total values. Commonly the higher flow rates with the same temperature boundary conditions provide lower overestimation. That is clear, because for the introduced case the Reynolds number in some areas decrease significantly to the boarder of laminar flow. And the k-epsilon is typical model for high turbulent flow, but it is relative robust. A typical problem is the entrance of the heat exchanger. The gap between two fins is relative small, so the flow is forced to laminar, but at the entrance to the zone is a relative high turbulent component, which dissipates fast. For that reason it is not possible to define the heat exchanger as a laminar zone.

For similar future computations should be refined the zone behind the air grill, if the velocity profile should be measured in a similar length, but for the heat transfer the influence is marginal. The heat exchanger zone should be probably divided in a turbulent and laminar zone to improve the heat transfer prediction. The turbulence zone should be located in the heat exchanger air entrance.

\section{References}

1. J. H. Ferziger, M. Perić, Computational Methods for Fluid Dynamics, (Springer-Verlag, Berlin, 2002)

2. J. Př́íhoda, P. Louda, Matematické modelování proudění, (ČVUT, Prague, 2007)

3. T. KuppanHeat Exchanger Design Handbook,(Taylor \& Francis Group, Boca Raton, 2013) 